\section{Le code de la route des axones se complique}

Vieri Failli, Thomas di Meglio, Alain Chédotal
Équipe Développement neuronal, CNRS UMR7102, Université Paris 6, 9, quai Saint-Bernard, 75005 Paris, France.

chedotal@infobiogen.fr

seule fois. Des recherches génétiques menées chez la drosophile ont conduit à la découverte de plusieurs molécules contrôlant le comportement des axones vis-à-vis de la ligne médiane. Ces travaux ont montré que les cellules de la ligne médiane sécrètent une protéine appelée Slit qui, en se fixant sur son récepteur Roundabout (Robo), ancré à l'extrémité des axones en croissance, va les contraindre à s'éloigner de la ligne $(\rightarrow) \mathrm{m} / \mathrm{s}$ médiane $(\rightarrow)$.

Chez les mammifères, y com-

$1999, n^{\circ} 6-7$, névraxe en s'entrecroisant avec les formations similaires, controlatérales. II existe trois zones principales de décussation: la protubérance annulaire, les pédoncules cérébraux et la zone antérieure de la moelle épinière. C'est au cours de cette décussation que les faisceaux nerveux franchissent la ligne médiane et deviennent controlatéraux par rapport à leur point d'origine. pris chez l'homme, on connaît trois récepteurs Robo (Robo-1-3) et trois protéines ligne médiane. Chez les vertébrés, la

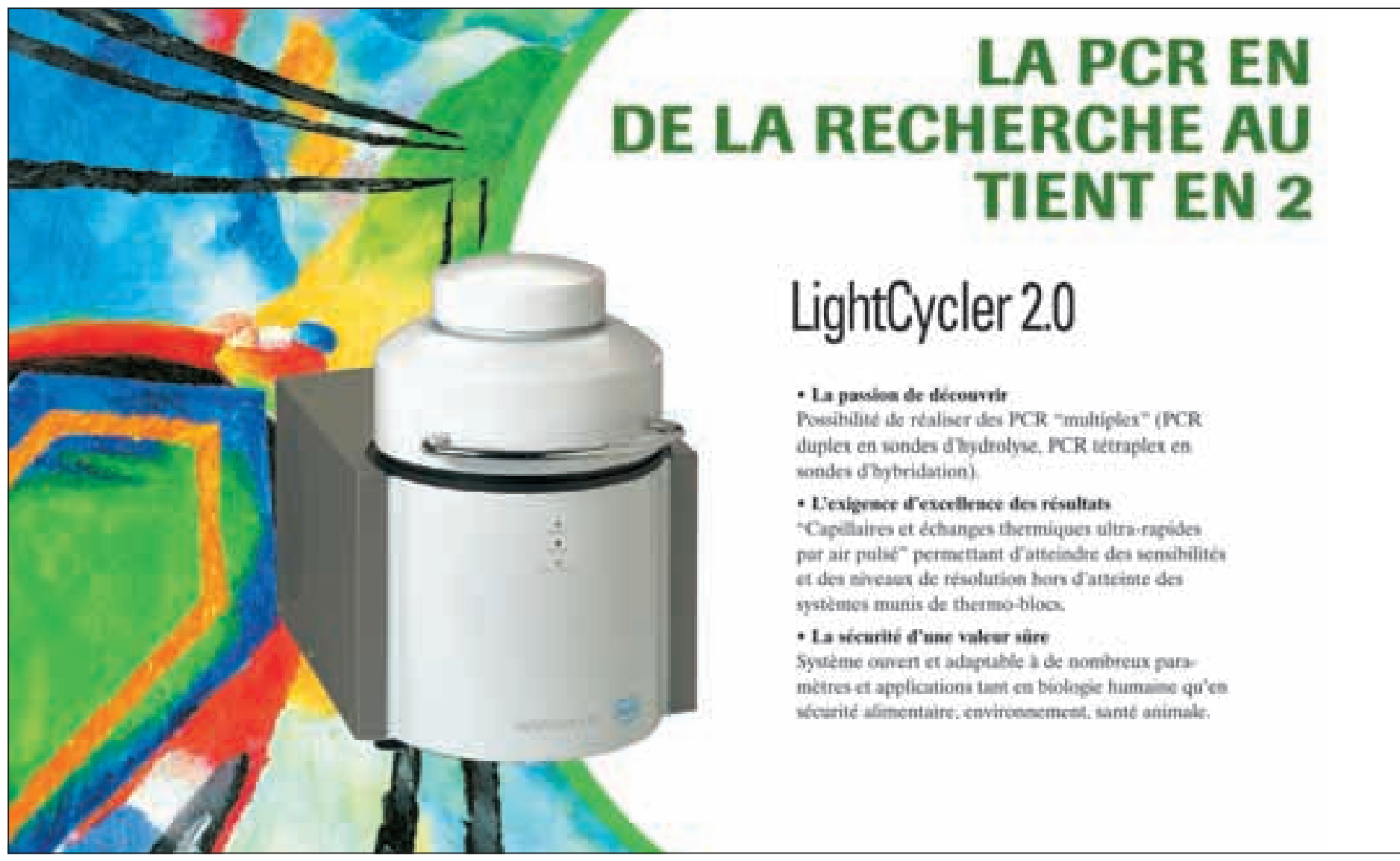


Slit (Slit-1-3), toutes exprimées au niveau de la ligne médiane du système nerveux mais aussi dans d'autres organes comme le poumon et le rein. Des expériences, faisant notamment appel à la culture cellulaire, ont montré que l'activité répulsive des molécules Slit pour les axones exprimant Robo est également conservée au cours de l'évolution. On a également observé que ces molécules pouvaient influencer la migration des neurones, des leucocytes $(\rightarrow)$ et de certaines cellules tumo-

$(\rightarrow) \mathrm{m} / \mathrm{s}$ 2002, $n^{\circ} 11$, p. 1075 rales ainsi que la croissance des vaisseaux sanguins (angiogenèse) [1]. Un pas important vers la confirmation du rôle essentiel des Slit et des Robo dans le développement du système nerveux des mammifères a été fait récemment grâce à l'étude de plusieurs lignées de souris modifiées génétiquement par la technique de recombinaison homologue de façon à ne plus synthétiser une ou plusieurs de ces molécules Slit ou leurs récepteurs Robo. En l'absence de Slit, l'organisation des projec- tions est fortement perturbée dans l'ensemble du système nerveux [2-5]. D'une manière générale, des axones qui, chez les souris normales, évitent la ligne médiane ont un comportement opposé chez les souris déficientes en Slit et traversent cette dernière. Une autre information tirée de l'analyse de ces mutants est l'extrême redondance fonctionnelle des Slit. Ainsi, les projections des neurones du bulbe olfactif, qui poussent latéralement à la surface du cerveau, à distance de la ligne médiane, sont normales chez les souris déficientes en Slit-1 ou en Slit-2. En revanche, ces projections sont désorganisées lorsque l'on inactive chez une même souris les gènes codant pour Slit-1 et Slit-2 [3]. De même, dans la moelle épinière, l'absence de Slit-1, ou de Slit-2 et même simultanément de Slit-1 et de Slit-2 n'empêche pas les axones des neurones commissuraux de croiser la plaque du plancher. Ce n'est que lorsque les trois molécules Slit sont absentes que les projections commissurales se développent anormalement [5]. Que se passe-t-il en l'absence des récepteurs Robo? À ce jour, seul le phénotype des souris dont un seul gène Robo a été inactivé a été analysé et, pour deux d'entre elles, seulement au niveau de la moelle épinière $[5,6]$. Les neurones commissuraux médullaires expriment les trois récepteurs Robo à l'approche de la plaque du plancher. Comme chez la drosophile, l'expression de Robo-l et de Robo-2 augmente fortement une fois la plaque du plancher croisée. De manière plus surprenante, l'expression de Robo-3 (aussi appelé Rig-1) s'estompe peu après la décussation ${ }^{1}$ [6]. L'analyse des souris mutantes pour Robo-1 montre que de nombreux axones commissuraux s'arrêtent au niveau de la ligne médiane sans pouvoir la quitter, comme chez les souris déficientes en Slit, ce qui confirme que c'est bien l'interaction Slit/Robo qui permet aux axones de s'éloigner de la plaque du plancher [6]. En revanche, le développement des axones commissuraux apparaît relativement normal chez les souris déficientes en Robo-2. La formation d'autres tissus est cependant

\section{TEMPS RÉEL DIAGNOSTIC MÉDICAL SYSTĖMES}

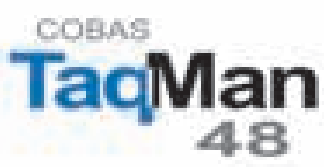

* f" et seil sysicme de PCR ea iemps reel pour le slagnoutic de ruutine.

- Systeme et réactifs muryues CZIVD pour la quantificatice HIV, HCV er HBV.

- Perlormaraces incrales en lincarite et scuils de scmitilite evocptionnela.

- Diagnoatic PCAt rendu en 2.3 herares.

\section{MARQUAGE} CE-IVD

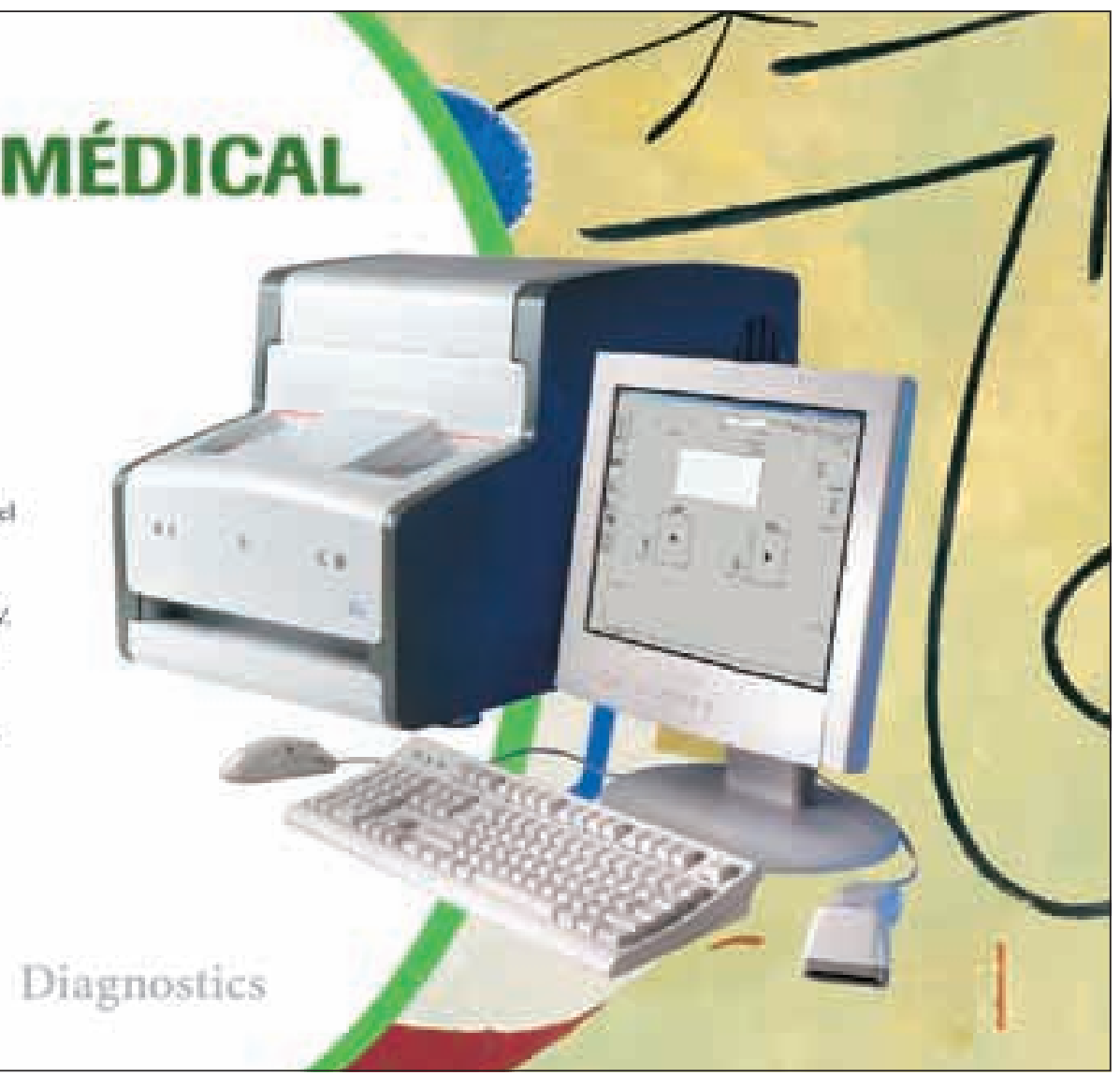


affectée, notamment celle du rein qui se développe anormalement en l'absence de Robo-2 [7]. C'est cependant des souris déficientes en Robo-3 que sont venus les résultats les plus inattendus: les axones commissuraux de la moelle épinière ne sont plus capables de croiser la plaque du plancher [6]. Qu'en est-il des autres axones commissuraux du cerveau? Notre équipe a étudié plusieurs populations de neurones du tronc cérébral qui envoient des axones dans le cervelet, d'où leur nom de neurones précérébelleux, et ont un rôle important dans le contrôle des mouvements et de l'équilibre [8]. Tous ces neurones projettent leurs axones à travers la plaque du plancher. En outre, pendant le développement, le corps cellulaire de certains neurones précérébelleux va aussi migrer à travers la ligne médiane. Nous avons montré que tous les neurones précérébelleux expriment fortement Robo-3/Rig-1 jusqu'à ce que leurs axones ou leurs corps cellulaires aient croisé la ligne médiane. Chez les souris déficientes en Robo-3, ni les axones des neurones précérébelleux, ni leurs corps cellulaires ne traversent la plaque du plancher [8]. Le phénotype est donc identique à celui qui est observé pour les axones de la moelle épinière. Ces résultats sont surprenants car ils font de Robo-3 un régulateur négatif de l'activité inhibitrice des Slit, ce qui est inhabituel pour un récepteur Robo classique. Le mode d'action de Robo-3 reste hypothétique, mais l'on pense qu'il pourrait bloquer l'activation par Slit des autres récepteurs Robo. La concentration élevée de Robo-3 sur les axones qui s'approchent de la ligne médiane rendrait ces derniers insensibles à l'action répulsive des Slit. Une fois celle-ci franchie, l'extinction rapide de l'expression de Robo-3 permettrait l'activation des autres récepteurs Robo, contraignant ainsi les axones à s'éloigner de la ligne médiane. Chez les souris déficientes en Robo-3, les axones sont repoussés prématurément et ne peuvent approcher la ligne médiane. Une autre surprise de taille est venue de l'étude génétique d'un syndrome humain rare [9], associant une paralysie des mouvements oculaires horizontaux à une forte scoliose (horizontal gaze palsy with progressive scoliosis). Chez ces patients, des analyses d'imagerie cérébrale par RMN (résonance magnétique nucléaire) ont montré une atrophie du noyau abducens, dont les neurones moteurs contrôlent les muscles oculaires responsables des déplacements horizontaux de l'œil. Ces travaux ont aussi mis en évidence une forte atrophie d'un groupe de neurones précérébelleux constituant les noyaux du pont. Enfin, l'analyse de la conduction des informations sensorielles ascendantes proprioceptives (toucher) et des informations motrices descendantes (faisceau corticospinal) a montré que, chez les patients atteints, les axones transmettant ces informations ne traversent pas la ligne médiane contrairement aux individus normaux. L'analyse génétique de dix familles de patients a révélé que tous sont porteurs de mutations dans le gène codant pour l'homologue humain de Robo-3. Les patients sont tous homozygotes pour l'allèle muté et les individus hétérozygotes sont normaux. Ces résultats confirment donc que l'expression de Robo-3 par les neurones commissuraux est nécessaire au croisement de la ligne médiane et que cette fonction est conservée dans l'évolution. $\diamond$

Axone guidance at the midline gets more complex

\section{RÉFÉRENCES}

1. Wang B, Xiao Y, Ding BB, et al. Induction of tumor angiogenesis by Slit-Robo signaling and inhibition of cancer growth by blocking Robo activity. Cancer Cell 2003; 4: 19-29.

2. Bagri A, Marin 0, Plump AS, et al. Slit proteins prevent midline crossing and determine the dorsoventral position of major axonal pathways in the mammalian forebrain. Neuron 2002; 33: 233-48.

3. Nguyen-Ba-Charvet KT, Plump AS, Tessier-Lavigne M, Chedotal A. Slitl and Slit2 proteins control the development of the lateral olfactory tract. J Neurosci 2002; 22: 5473-80.

4. Plump AS, Erskine L, Sabatier C, et al. Slitl and Slit2 cooperate to prevent premature midline crossing of retinal axons in the mouse visual system. Neuron 2002; 33: 219-32.

5. Long H, Sabatier C, Ma L, et al. Conserved roles for slit and robo proteins in midline commisural axon guidance. Neuron 2004; 42: 213-23.

6. Sabatier C, Plump AS, Ma L, et al. The divergent Robo family protein Rig-1/Robo3 is a negative regulator of slit responsiveness required for midline crossing by commissural axons. Cell 2004; 117: 157-69.

7. Grieshammer U, Le MA, Plump AS, et al. Slit2-mediated Robo 2 signaling restricts kidney induction to a single site. Dev Cell 2004; 6: 709-17.

8. Marillat V, Sabatier C, Failli V, et al. The Slit receptor Rig-1/Robo3 controls the development of precerebellar neurons. Neuron 2004; 43: 69-79.

9. Jen JC, Chan WM, Bosley TM, et al. Mutations in a human ROBO gene Disrupt hindbrain axon pathway crossing and morphogenesis. Science 2004; 304: 1509-13.

\section{NOUVELLE}

\section{Trois brins d'ADN dans le centre catalytique des ADN polymérases}

Patrick Lestienne

> Depuis plus de 40 ans, on admet que les ADN polymérases ne contiennent que deux brins d'ADN dans leur centre actif : le brin modèle et le brin en cours de synthèse.
Nous montrons dans ce travail qu'à partir d'une

Laboratoire de pharmacologie des agents anticancéreux, FRE 2618 CNRS, Institut Bergonié, 229, cours de l'Argonne, 33076 Bordeaux, France. lestienne@bergonie.org amorce formant une triple hélice, il est possible de répliquer de l'ADN en double brin si une certaine instabilité est locali- sée à proximité du site d'initiation de la réplication. Par conséquent, les ADN polymérases peuvent accomoder transitoirement trois brins d'ADN au lieu de deux comme cela est communément admis. Les conséquences portent à la fois sur les systèmes de réparation, voire des mécanismes de réarrangements de I'ADN. Des hypothèses évolu- 